\title{
MicroRNA-25/ATXN3 interaction regulates human colon cancer cell growth and migration
}

\author{
DINGYUN LI, TAO ZHANG, JIAJUN LAI, JIAN ZHANG, TING WANG, \\ YAFEI LING, SHENGQUAN HE and ZHIWEI HU
}

\begin{abstract}
Department of Gastrointestinal Surgery, Yue Bei People's Hospital, Shaoguan, Guangdong 512026, P.R. China
\end{abstract}
Received December 6, 2017; Accepted September 6, 2018

DOI: $10.3892 / \mathrm{mmr} .2019 .10090$

\begin{abstract}
The present study aimed to investigate the function of microRNA-25 (miR-25) in human colon cancer cell viability and migration in addition to the underlying possible mechanisms. miR-25 expression was upregulated in patients with colon cancer compared with the control group. Reverse transcription-quantitative polymerase chain reaction and gene chip technology were used to analyze the alterations of miR-25 in patients with colon cancer. Cell viability and cell migration were analyzed using MTT and wound healing assays, respectively, apoptosis was analyzed using flow cytometry, and western blot analysis was conducted to determine the protein expression of ataxin-3 (ATXN3), apoptosis regulator Bax (Bax) and cyclin D1. Overexpression of miR-25 increased cell viability and migration, decreased apoptosis, decreased caspase-3/9 activity level in addition to decreased Bax protein expression, and increased cyclin D1 protein expression in colon cancer cells. Furthermore, miR-25 was demonstrated to target ATXN3 and suppress ATXN3 protein expression. Downregulation of miR-25 induced apoptosis of colon cancer cells via increased expression ATXN3. Small interfering-ATXN3 inhibited the anti-cancer effects of miR-25 downregulation in colon cancer. Collectively, the present results demonstrated that miR-25 promoted human colon cancer cell viability and migration by regulating ATXN3 expression.
\end{abstract}

\section{Introduction}

Colorectal cancer (CRC) is a common malignant tumor with mortality ranking third among tumors. A recent study reported that CRC mortality in European and American developed countries is decreased (1). However, the morbidity and mortality of CRC in China remains high (1). The national standard of living is improving at present, along with accelerated population

Correspondence to: Dr Dingyun Li, Department of Gastrointestinal Surgery, Yue Bei People's Hospital, 133 South Huimin Road, Shaoguan, Guangdong 512026, P.R. China E-mail: bpwt73093@126.com

Key words: microRNA-25, ataxin-3, colon cancer, cell growth, migration aging and altered dietary structure (2). These factors have resulted in markedly increased morbidity of CRC in China, severely threatening human health (2). A study suggested that morbidity of CRC, particularly that of distal colon cancer and rectal cancer, increases gradually. Furthermore, it demonstrates a younger trend (3). The pathogenesis of CRC has not been completely elucidated (3). However, existing data suggest that $\mathrm{CRC}$ incidence is closely associated with a number of factors, including high fat/low cellulose diet, chronic colitis, colorectal adenoma and heredity (3). Other factors, including bilharziasis, pelvic radiation, environmental factor and smoking, may additionally affect CRC incidence (3). The pathogenesis of CRC remains unclear, with no early symptoms observed (3). This has contributed to the difficulties in the early diagnosis and treatment of CRC (1).

MicroRNA (miRNA) is a class of endogenous small non-coding RNA molecules, 22 nucleotides in length (4). It is the single-strand endogenous non-coding RNA that is extensively distributed in eukaryotes (5). The majority of miRNAs are transcribed by RNA polymerase II. The primary transcription product is called pri-miRNA. Pri-miRNA forms the mature miRNA following several steps of intranuclear and intracytoplasmic processing. miRNAs directly bind with the 3'-untranslated region (UTR) of target mRNAs to exert regulatory effect (6).

Virulence gene ataxin-3 (ATXN3) is located in the short arm of chromosome 14 . Its 3'-terminal protein-coding region contains the cytosine-adenine-uracil trinucleotide repetitive sequence (7). The repeat frequency is 12-40 in healthy subjects, and can reach $51-86$ in patients with CRC. The ATXN3 gene contains the abnormally amplified CAG repetitive sequence, which encodes ataxin-3 containing an expanded poly-glutamine (polyQ) peptide chain (8). This is a toxic protein that is extensively expressed in the human body $(8,9)$. It may selectively aggregate in specific regions of the nervous system, including the cerebellum, brainstem and spinal cord, to form neuronal intranuclear inclusion and may induce cell death $(7,9)$. The present study aimed to examine the function of miRNA-25 (miR-25) on human colon cancer cell viability and migration, and its potential mechanisms.

\section{Materials and methods}

Patients with CRC and survival rate. Serum samples from patients with CRC $(n=48)$ and healthy volunteers $(n=8)$ were 
collected at the Department of Gastrointestinal Surgery, Yue Bei People's Hospital (Shaoguan, China) between June 2011 to November 2011. The exclusion criteria for patients included any history of inflammatory bowel disease. Characteristics of patients with CRC and healthy volunteers are presented in Table I. Serum samples were collected and centrifuged at $1,000 \mathrm{xg}$ for $10 \mathrm{~min}$ at $4^{\circ} \mathrm{C}$. Every 3 months, patients were contacted by telephone for a follow-up period of 5 years and the results were measured using a log rank test. The present study was approved by the Ethics Committee of the Yue Bei People's Hospital. Written informed consent was obtained from all patients.

Reverse transcription-quantitative polymerase chain reaction $(R T-q P C R)$. Total RNA was extracted from the samples using TRIzol ${ }^{\circledR}$ reagent (Invitrogen; Thermo Fisher Scientific, Inc., Waltham, MA, USA) and cDNA synthesis was conducted with RevertAid first strand cDNA synthesis kit (Thermo Fisher Scientific, Inc.), according to the manufacturer's instructions. qPCR was conducted using a SYBR-Green Master Mix (Takara Biotechnology Co., Ltd., Dalian, China) and a CFX-96 qPCR system (Bio-Rad Laboratories, Inc., Hercules, CA, USA). The primers were as follows: miR-25-3p, forward: 5'-CATTGCACTTGTCTCGGTCTGA-3', reverse: 5'-GCT GTCAACGATACGCTACGTAACG-3' and U6, forward: 5'-CTCGCTTCGGCAGCACA-3', reverse: 5'-AACGCTTCA CGAATTTGCGT-3'. The thermocycling conditions were as follows: Incubation at $95^{\circ} \mathrm{C}$ for $5 \mathrm{~min}$, followed by 40 cycles of $95^{\circ} \mathrm{C}$ for $15 \mathrm{sec}, 60^{\circ} \mathrm{C}$ for $30 \mathrm{sec}$ and $72^{\circ} \mathrm{C}$ for $30 \mathrm{sec}$. The relative expression of miR-25 was calculated using the $2^{-\Delta \Delta C q}$ method (10). Higher miR-25 expression was defined as $>2$-fold the mean miR-25 expression of healthy volunteers and lower miR- 25 expression was defined as $\leq 2$-fold the miR- 25 expression of healthy volunteers.

Gene chip technology. A total of 500 ng RNA was hybridized to the SurePrint G3 Mouse Whole Genome GE K Microarray G4852A platform (G4852A; Agilent Technologies, Inc., Santa Clara, CA, USA). The data was quantified using Agilent Feature Extraction software version10.7.3.1 (Agilent Technologies, Inc.).

Cell culture and transfection. The human HCT116 colon cancer cell line was obtained from the Shanghai Cell Bank of Chinese Academy of Sciences (Shanghai, China) and cultured in RPMI-1640 medium (Invitrogen; Thermo Fisher Scientific, Inc.) supplemented with $10 \%(\mathrm{v} / \mathrm{v})$ fetal bovine serum (FBS; Gibco; Thermo Fisher Scientific, Inc.) at $37^{\circ} \mathrm{C}$ and $5 \% \mathrm{CO}_{2}$. miR-25 (5'-GGCCAGTGTTGAGAGGCA-3' and 5'-TGA CAGTGCCGGCC-3'), anti-miR-25 (5'-CTCCCTCACAGG ACAGCTGAACAC-3' and 5'-CTGCCCCCCCACATCTGC AGT-3') and negative mimics (5'-CCCCCCCCCCCCC-3' and 5'-CCCCCCCCCCCCC-3') were purchased from Sangon Biotech Co., Ltd. (Shanghai, China). In total, $100 \mathrm{nM}$ miR-25, anti-miR-25 and negative mimics were transfected into cells using the X-tremeGENE small interfering RNA (si)RNA transfection reagent (Roche Molecular Diagnostics, Pleasanton, CA, USA). A total of 100 ng si-ATXN3 (sc-40359; Santa Cruz Biotechnology; sequence is commercially unavailable) was transfected into cells using the X-tremeGENE siRNA transfection reagent (Roche Molecular Diagnostics) for $48 \mathrm{~h}$.
Table I. Characteristics of patients with CRC and healthy volunteers.

\begin{tabular}{lcc}
\hline Characteristic & CRC $(\mathrm{n}=72)$ & Healthy $(\mathrm{n}=8)$ \\
\hline Age, years & & \\
$\leq 60$ & 29 & 4 \\
$>60$ & 43 & \\
Sex & & 3 \\
Female & 25 & 5 \\
Male & 47 & \\
Tumor size, cm & & - \\
$\leq 3.0$ & 31 & - \\
$>3.0$ & 41 & - \\
TNM staging system & & - \\
I & 8 & - \\
II & 15 & \\
III-IV & 49 & \\
\hline
\end{tabular}

CRC, colorectal cancer; TNM, tumor-node-metastasis.

Cell viability analysis and cell migration assay. Cells were harvested at $0,24,48$ and $72 \mathrm{~h}$ after transfection. A total of $20 \mu 1$ MTT solution (5 mg/ml; Sigma-Aldrich; Merck KGaA, Darmstadt, Germany) was added to the cells and cultured for $4 \mathrm{~h}$ at $37^{\circ} \mathrm{C}$. The supernatant was removed using a pipette, and $150 \mu \mathrm{l}$ dimethyl sulfoxide was added to the cells and cultured for $20 \mathrm{~min}$ at $37^{\circ} \mathrm{C}$. Optical density was measured at $562 \mathrm{~nm}$ using a $\mu$ Quant $^{\mathrm{TM}}$ Microplate Spectrophotometer (BioTek Instruments, Inc., Winooski, VT, USA).

Cells $\left(1 \times 10^{4}\right.$ cell $\left./ \mathrm{ml}\right)$ at $24 \mathrm{~h}$ after transfection were cultured in $100 \mu 1$ media without serum in the upper chamber of Millicells (pore size $8 \mu \mathrm{m}$; EMD Millipore, Billerica, MA, USA) at $37^{\circ} \mathrm{C}$ for $24 \mathrm{~h}$. The lower chamber contained RPMI-1640 medium with 20\% FBS. The migrated cells were fixed with $70 \%$ ethanol at room temperature for $30 \mathrm{~min}$, stained with $5 \mathrm{mg} / \mathrm{ml}$ crystal violet solution for $30 \mathrm{~min}$ at room temperature and counted under a confocal microscope (Leica SP5; Leica Microsystems, Inc., Buffalo Grove, IL, USA).

Apoptosis rate analysis by flow cytometry. Cells at $48 \mathrm{~h}$ after transfection were washed twice with PBS, and $500 \mu 1$ cell binding buffer (BD Pharmingen, San Diego, CA, USA) was added. Cells were stained with $5 \mu \mathrm{l}$ Annexin V-PE conjugate and $5 \mu \mathrm{l}$ propidium iodide for $15 \mathrm{~min}$ in the dark. The apoptosis rate of the cells was subsequently determined using a flow cytometer (C6; BD Pharmingen, San Diego, CA, USA) and analyzed using Image Lab version 3.0 (Bio-Rad Laboratories, Inc.).

Western blot analysis. Total cell protein were collected and extracted $48 \mathrm{~h}$ after transfection using radioimmunoprecipitation assay buffer (Pierce; Thermo Fisher Scientific, Inc.) and quantified using a BCA Protein Concentration kit (P0009; Beyotime Institute of Biotechnology, Haimen, China). Total cell protein $(50 \mu \mathrm{g})$ was separated by SDS-PAGE on $10-12 \%$ gels and subsequently transferred to nitrocellulose membranes (Pall 

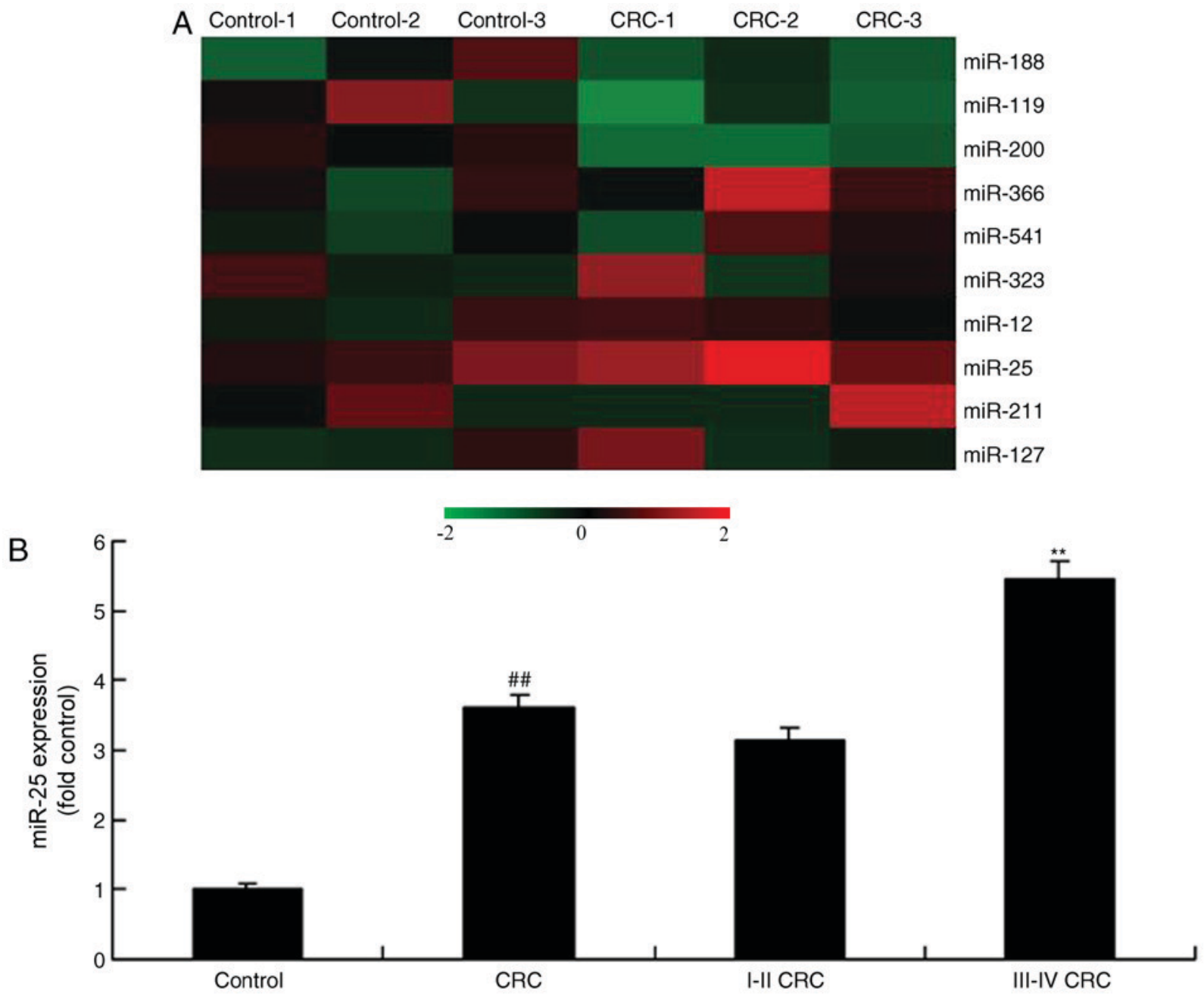

Figure 1. miR-25 expression in human colon cancer. (A) Gene chip heat map for miR expression and (B) miR-25 expression in human colon cancer. Data are presented as the mean \pm standard deviation. ${ }^{\# \#} \mathrm{P}<0.01$ vs. control (healthy volunteers); ${ }^{* *} \mathrm{P}<0.01$ vs. I-II CRC. miR, microRNA; control, healthy volunteer group; CRC, colorectal cancer.

Life Sciences, Port Washington, NY, USA). The membranes were incubated with ATXN3 (ab175265, 1:1,000, Santa Cruz Biotechnology, Inc., Dallas, TX, USA), cyclin D1 (sc-70899; 1:1,000; Santa Cruz Biotechnology, Inc.), apoptosis regulator Bax (Bax; sc-6236; 1:1,000; Santa Cruz Biotechnology, Inc.) and GAPDH (sc-293335; 1:5,000; Santa Cruz Biotechnology, Inc.) at $4^{\circ} \mathrm{C}$ overnight following blocking with $5 \%$ skimmed milk in Tris-buffered saline with Tween 20 (TBST) at room temperature for $2 \mathrm{~h}$. The membranes were washed for $10 \mathrm{~min}$ in TBST and incubated with goat anti-rabbit horseradish peroxidase-conjugated immunoglobulin G (sc-2004; 1:5,000; Santa Cruz Biotechnology, Inc.) for $1 \mathrm{~h}$ at $37^{\circ} \mathrm{C}$. Proteins were visualized using enhanced chemiluminescent reagent (Santa Cruz Biotechnology, Inc.) and analyzed using Image-Pro Plus version 6.0 software (Media Cybernetics, Inc., Rockville, MD, USA).

Caspase-3/9 activity. Total protein was extracted from cells using radioimmunoprecipitation assay buffer (Pierce; Thermo Fisher Scientific, Inc.) $48 \mathrm{~h}$ after transfection and quantified using BCA Protein Concentration kit (P0009; Beyotime Institute of Biotechnology). A total of $10 \mu \mathrm{g}$ protein was used to measure caspase-3/9 activity using
Caspase-3/9 activity kits (cat. nos. C1116 and C1158; Beyotime Institute of Biotechnology), according to the manufacturer's instructions. Optical density at $405 \mathrm{~nm}$ was measured using a $\mu$ Quant $^{\mathrm{TM}}$ Microplate Spectrophotometer (BioTek Instruments, Inc.).

Luciferase reporter assay. The partial sequence of the ATXN3'-UTR was cloned downstream of the firefly luciferase gene in the pGL3-Control Vector. HCT116 cell was transfected with ATXN3 and microRNA-25 or negative mimics using X-tremeGENE siRNA transfection reagent (Roche Molecular Diagnostics). The cells were harvested and assayed with the dual-luciferase assay (Promega, Madison, WI, USA) after $48 \mathrm{~h}$ of transfection. The results are expressed as the relative luciferase activity (Firefly LUC/Renilla LUC).

Statistical analysis. Experiments were repeated three times and the data are presented as the mean \pm standard deviation. Statistical analysis was performed using SPSS version 17.0 (SPSS, Inc., Chicago, IL, USA). Statistical differences between two groups were determined by Student's t-test. Statistical differences between three groups were determined by one-way analysis of variance and Tukey's post hoc test. $\mathrm{P}<0.05$ was 

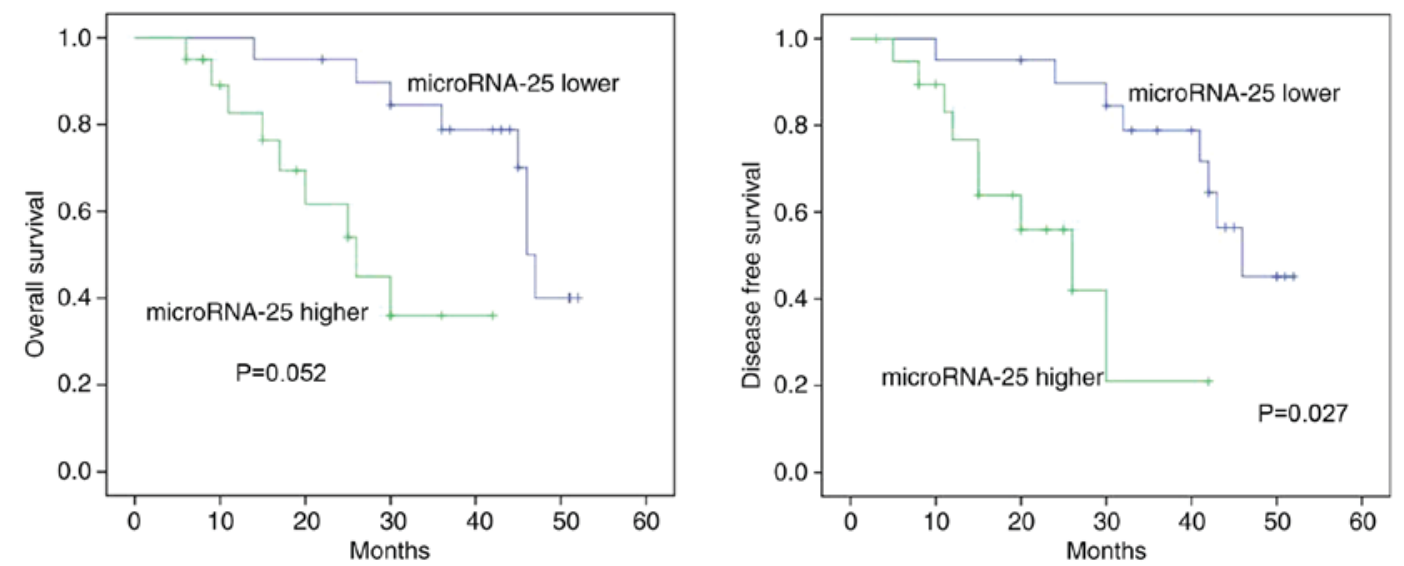

Figure 2. Relative microRNA-25 expression and overall survival/disease free survival in human colon cancer. Relative microRNA-25 expression and overall survival and disease free survival in human colon cancer.
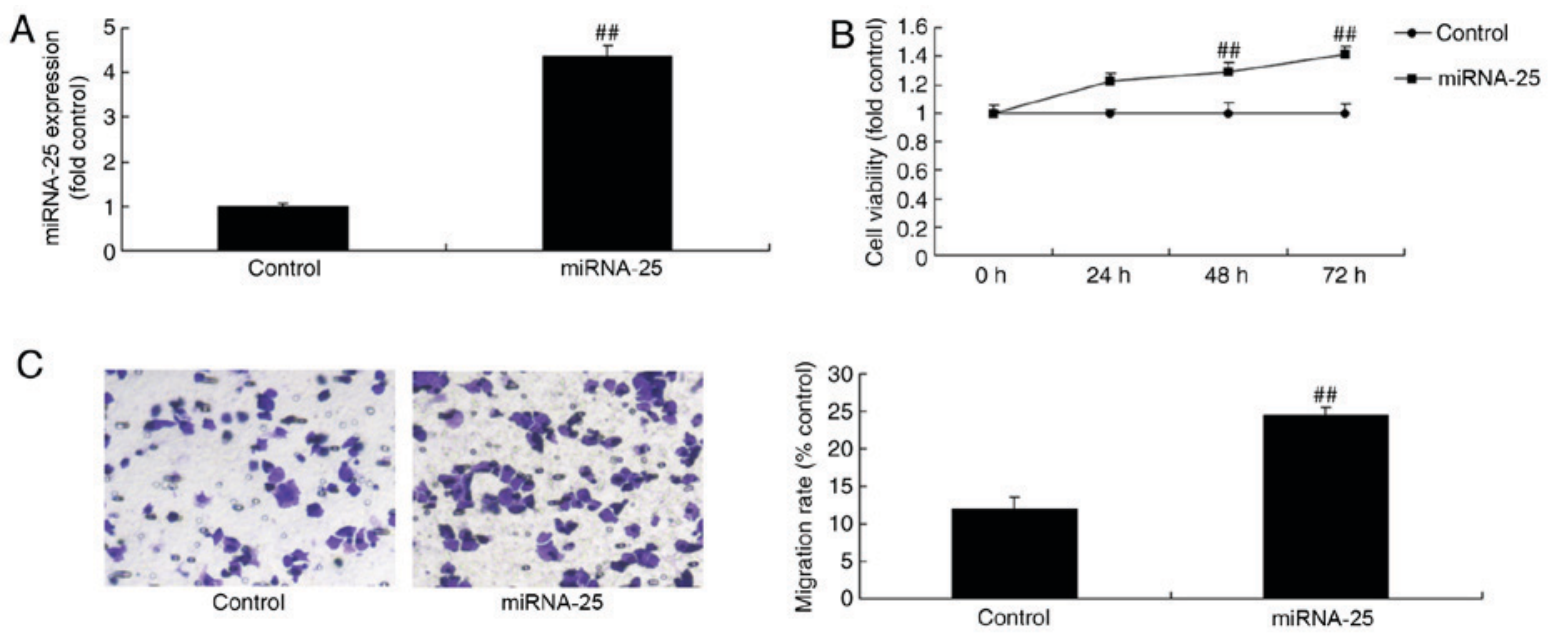

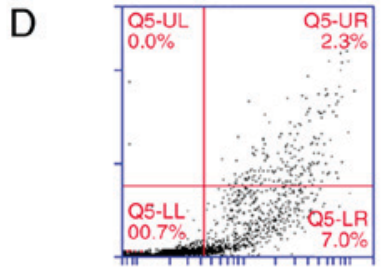

Control

E

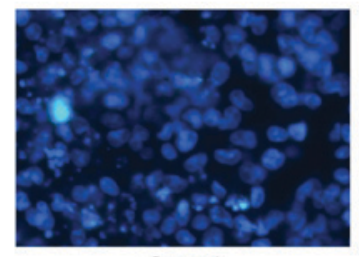

Control

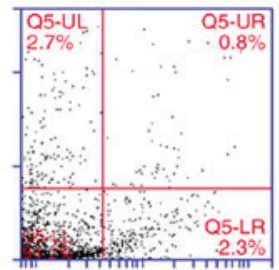

miRNA-25

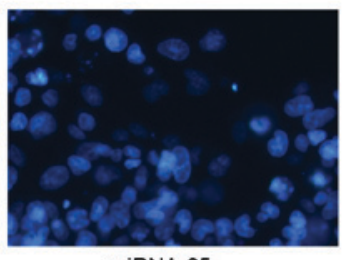

miRNA-25
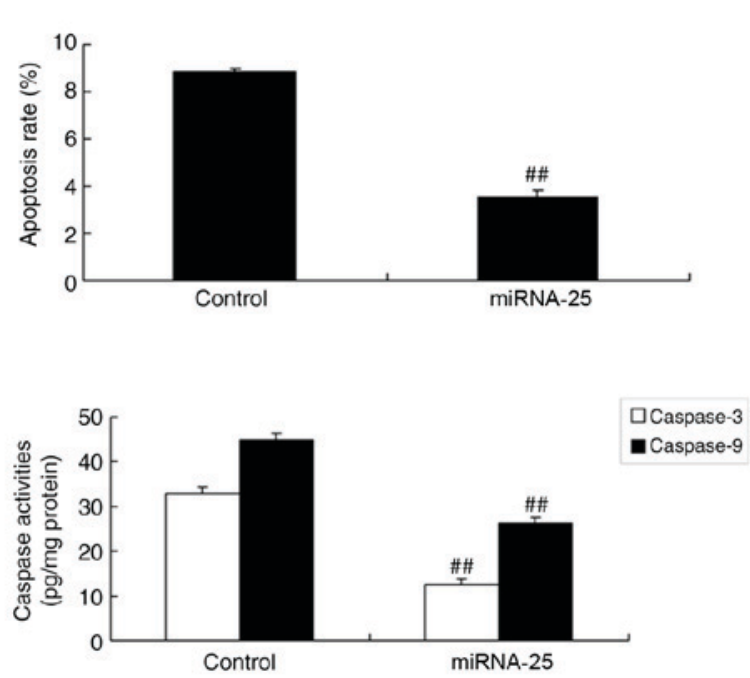

Figure 3. Overexpression of miRNA-25 promotes cell viability and migration of colon cancer cells. Cells were transfected with miR-25 mimics and (A) miRNA-25 expression, (B) cell viability, (C) cell migration (magnification, x100), (D) apoptosis rate and (E) caspase-3/9 activity level were determined. Data are presented as the mean \pm standard deviation. ${ }^{\#} \mathrm{P}<0.01$ vs. respective control. Control, negative control group; miRNA-25, microRNA-25; UL, upper left; UR, upper right; LL, lower left; LR, lower right.

considered to indicate a statistically significant difference. Overall survival (OS) and disease free survival (DFS) were analyzed using Kaplan-Meier survival curves.

\section{Results}

miR-25 expression in human colon cancer. The alterations 

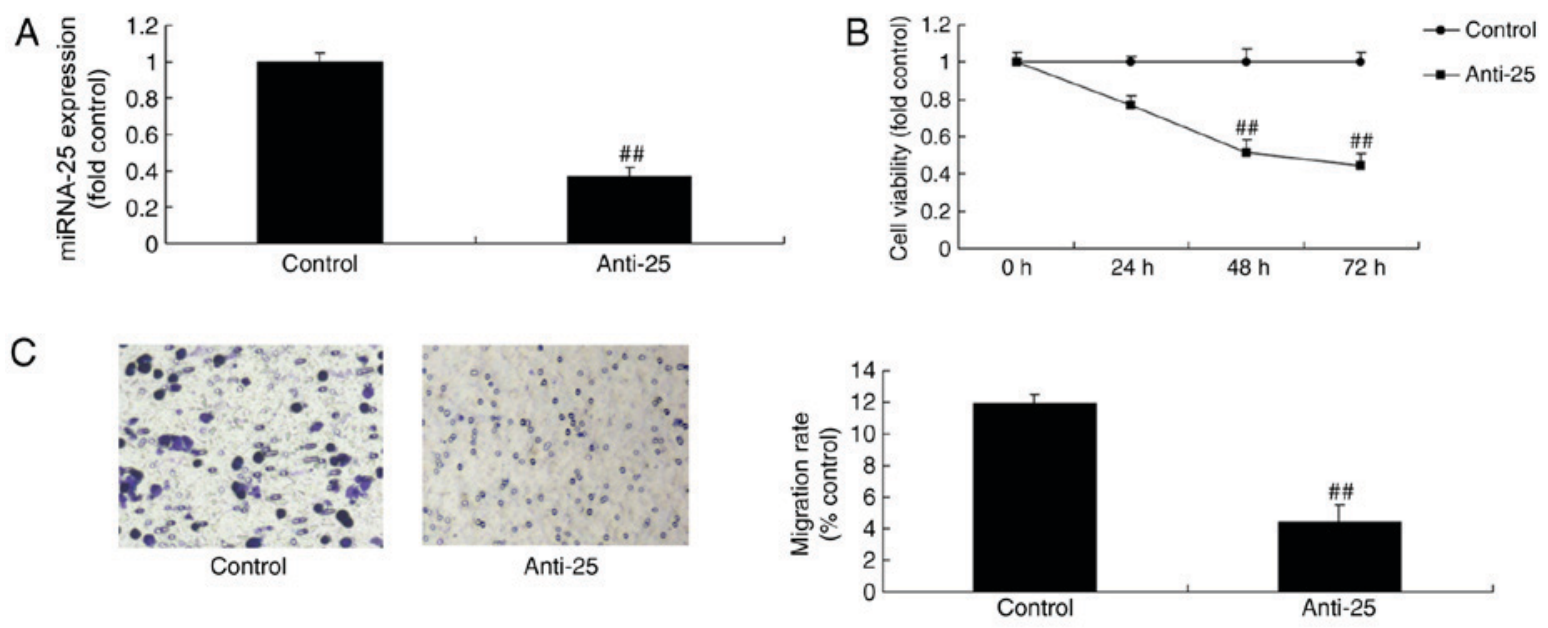

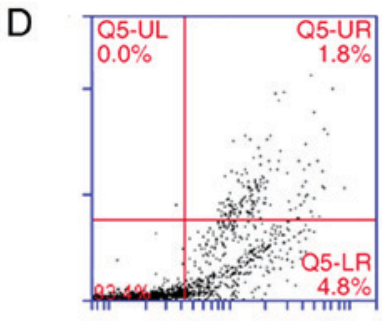

Control
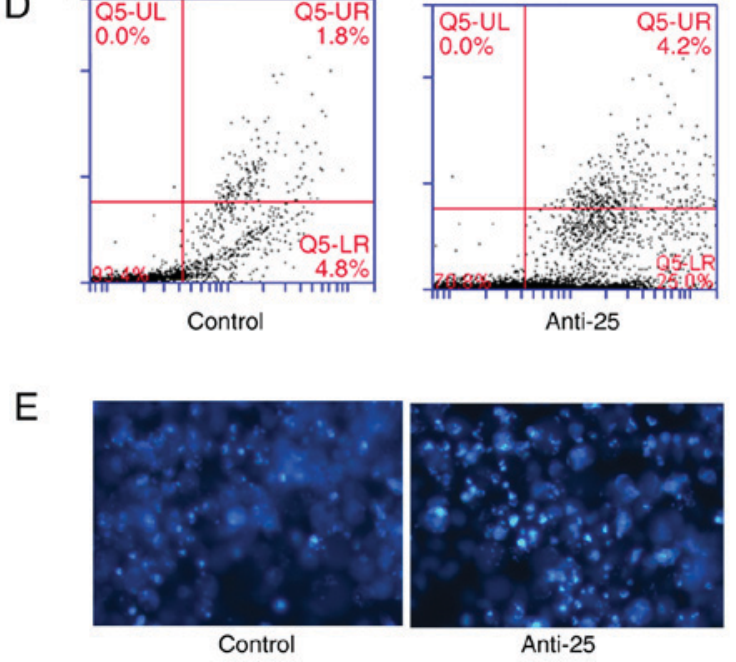

Anti-25
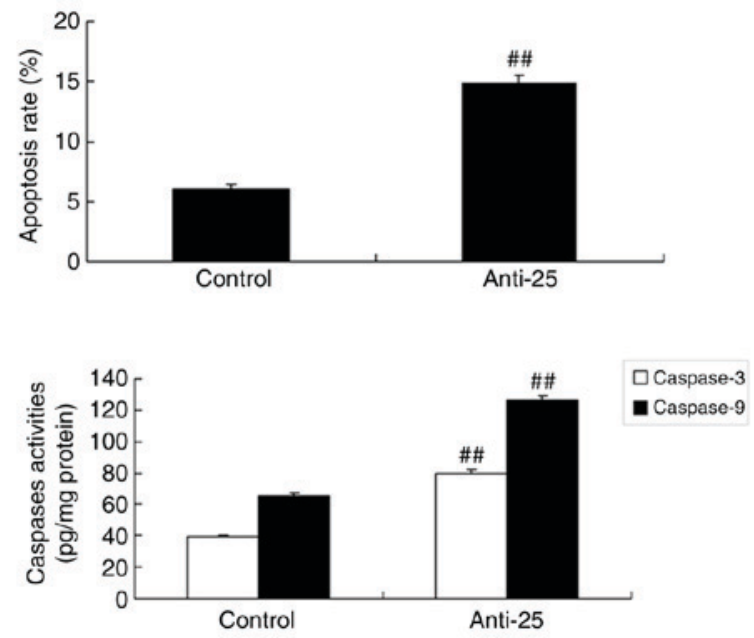

Figure 4. Downregulation of miRNA-25 reduces cell viability and migration of colon cancer cells. Cells were transfected with miR-25 inhibitor oligos and (A) miRNA-25 expression, (B) cell viability, (C) migration, (D) apoptosis rate and (E) caspase-3/9 activity level were determined. Data are presented as the mean \pm standard deviation. ${ }^{\# \#} \mathrm{P}<0.01$ vs. respective control. Control, negative control group; miRNA-25, microRNA-25; Anti-25, miR-25 inhibitor; UL, upper left; UR, upper right; LL, lower left; LR, lower right.

of miRNAs in human colon cancer tissue were examined. As demonstrated in Fig. 1, miR-25 expression was upregulated in patients with colon cancer, compared with the controls. In addition, the expression of miR-25 in the serum of patients with stage III-IV colon cancer was significantly increased compared with patients with stage I-II colon cancer (Fig. 1B).

miR-25 expression is associated with OS/DFS in human colon cancer. The association between miR-25 expression and OS/DFS in human colon cancer was analyzed. As demonstrated in Fig. 2, the OS $(\mathrm{P}=0.052)$ and DFS $(\mathrm{P}=0.027)$ of patients with human colon cancer with higher miR-25 expression were markedly lower compared with lower miR-25 expression.

Overexpression of miR-25 promotes cell viability and migration of colon cancer cells. To investigate whether the overexpression of miR-25 promoted cell viability and migration of colon cancer cells, colon cancer cells were transfected with miR-25 mimics. As a result, miR-25 expression was significantly upregulated in colon cancer cells by miR-25 mimics compared with the control group (Fig. $3 \mathrm{~A} ; \mathrm{P}<0.01$ ).
Furthermore, overexpression of miR-25 significantly increased cell viability and migration, decreased the apoptosis rate and decreased caspase-3/9 activity level of colon cancer cells compared with the control group (Fig. 3B-E; P<0.01).

Downregulation of $\mathrm{miR}-25$ decreases cell viability and migration of colon cancer cells. Additionally, the effects of miR-25 downregulation on cell viability and migration of colon cancer cells were examined. There was a significant inhibition of miR-25 expression in colon cancer cells transfected with miR-25 inhibitor compared with the control group (Fig. 4A; $\mathrm{P}<0.01)$. Downregulation of miR-25 significantly decreased cell viability and migration, and increased apoptosis and the caspase-3/9 activity level of colon cancer cell, compared with the control group (Fig. 4B-E; $\mathrm{P}<0.01$ ). Together, the present results demonstrated that $\mathrm{miR}-25$ regulated colon cancer cell growth; however, its potential mechanisms requires further investigation.

miR-25 targets ATXN3 in colon cancer cell. The possible mechanisms of miR-25 in colon cancer cells were 
A

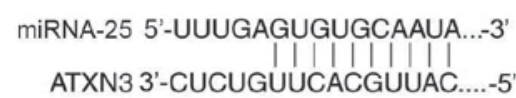

C

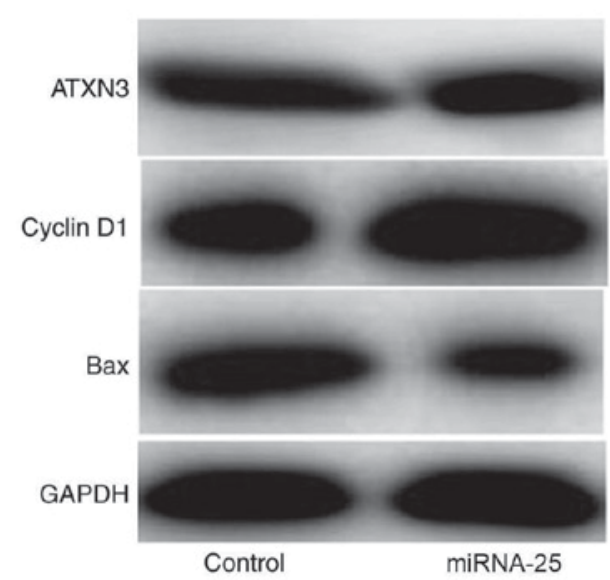

E

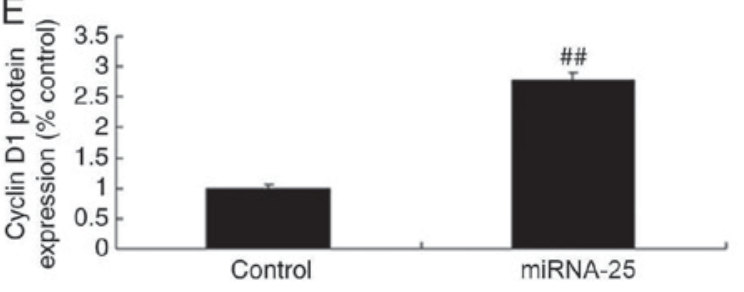

G

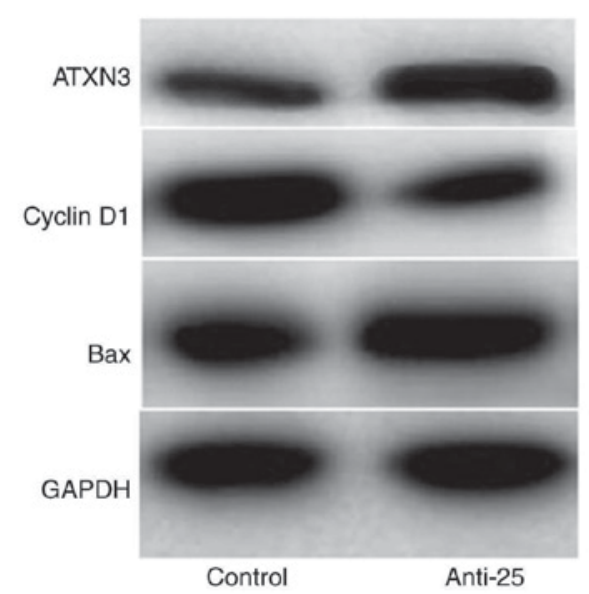

I

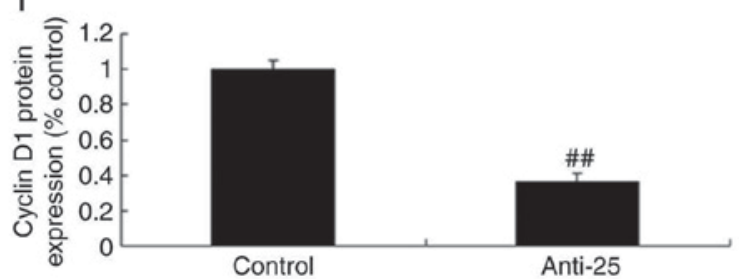

$\mathrm{B}$
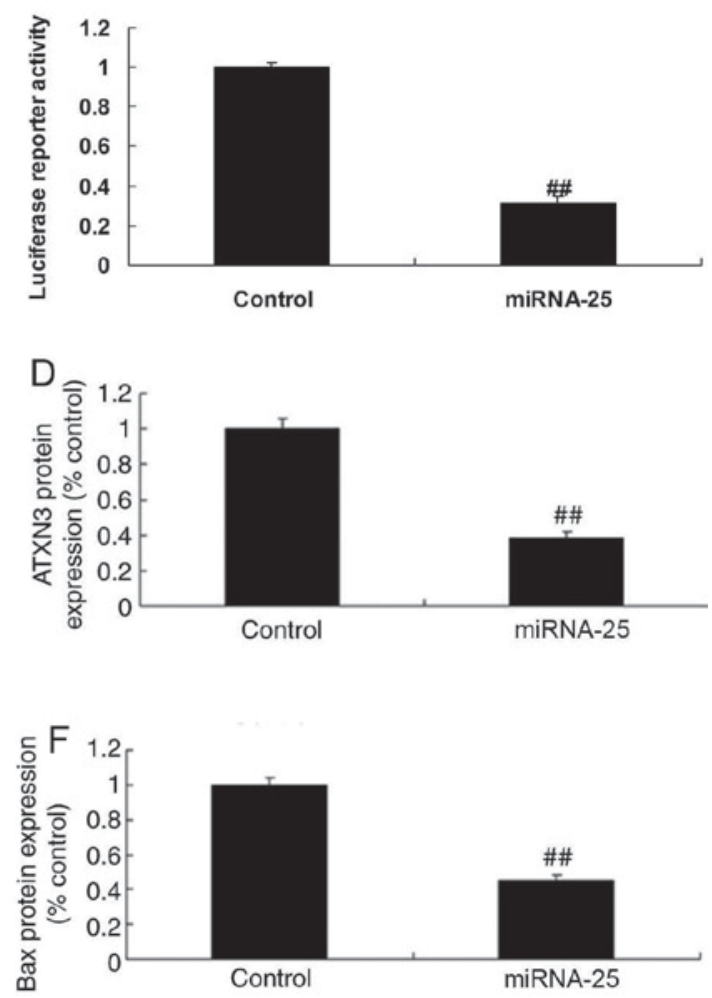

$\mathrm{H}$
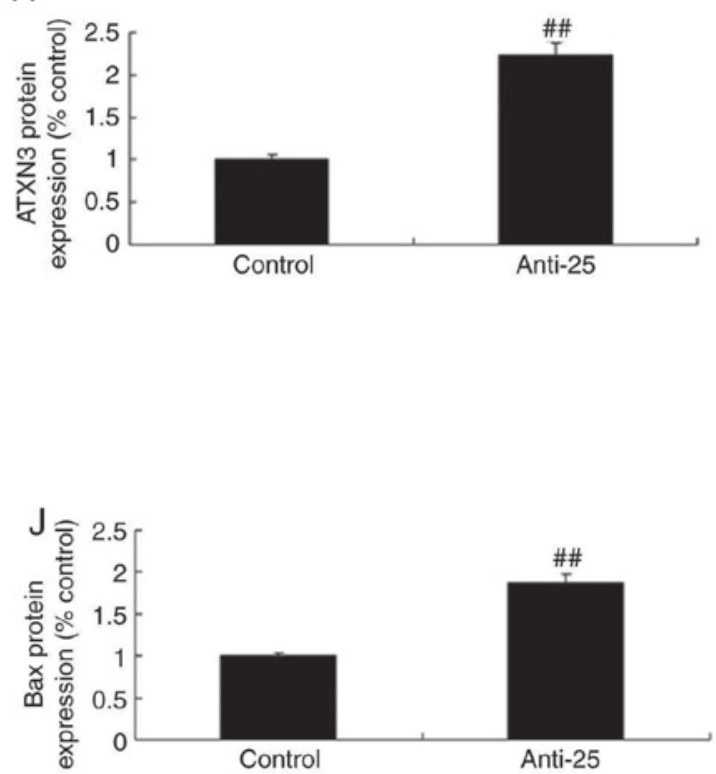

Figure 5. miRNA-25 targets ATXN3 in colon cancer cells. (A) Mutation analysis of the miR-25 binding site 3'-untranslated region of ATXN3. (B) Luciferase reporter activity. (C) Cells were transfected with miR-25 mimics and (D) ATXN3, (E) cyclin D1 and (F) Bax expression were determined by western blot analysis and densitometry. (G) Cells were transfected with miR-25 inhibitor oligos and (H) ATXN3, (I) cyclin D1 and (J) Bax expression were determined by western blot analysis and densitometry. Data are presented as the mean \pm standard deviation. ${ }^{\# \#} \mathrm{P}<0.01$ vs. respective control. Control, negative control group; miRNA-25, microRNA-25; Anti-25, miR-25 inhibitor; ATXN3, ataxin-3; Bax, apoptosis regulator Bax.

investigated. Mutation analysis revealed an miR-25 binding site in the 3'-UTR of ATXN3 (Fig. 5A) and overexpression of miR-25 reduced luciferase reporter activity levels when compared with negative group (Fig. 5B). Overexpression of miR-25 significantly decreased ATXN3 and Bax protein expression, and increased cyclin D1 protein expression 

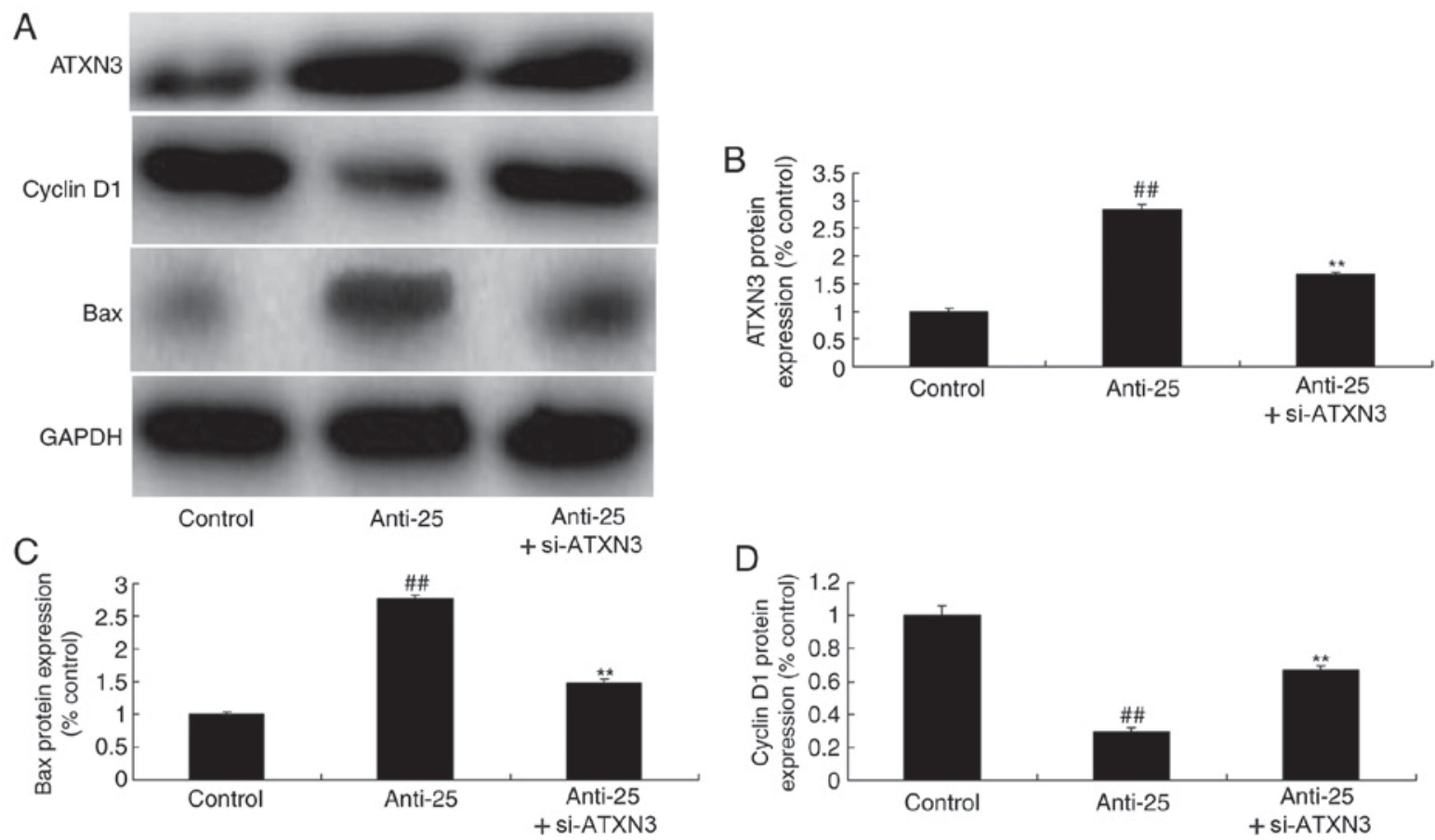

Figure 6. si-ATXN3 inhibits the anti-cancer effects of microRNA-25 downregulation on ATXN3 protein expression. (A) Western blot and densitometry of (B) ATXN3, (C) Bax and (D) cyclin D1 expression. Data are presented as the mean \pm standard deviation. ${ }^{\# /} \mathrm{P}<0.01$ vs. control; ${ }^{* *} \mathrm{P}<0.01$ vs. anti-25. Control, negative control group; Anti-25, microRNA-25 inhibitor; si, small interfering RNA; ATXN3, ataxin-3; Bax, apoptosis regulator Bax.

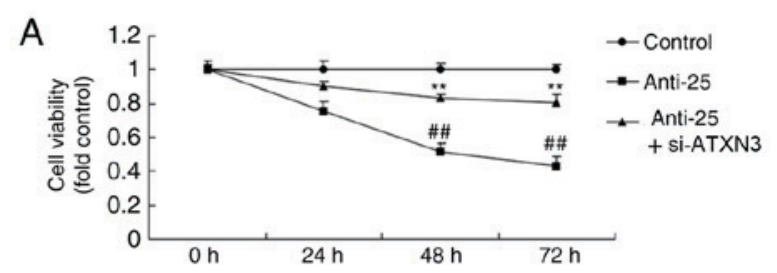

B

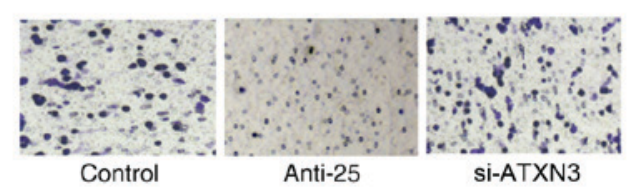

C

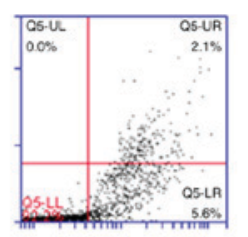

Control
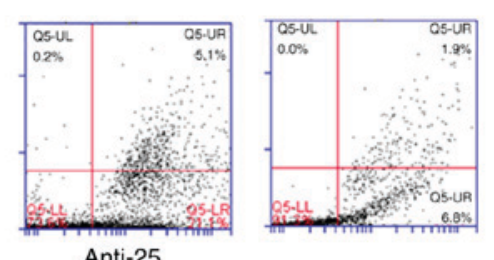

D

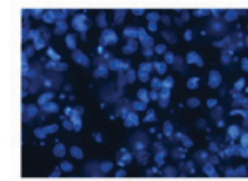

Control

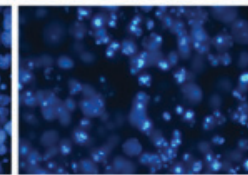

Anti-25

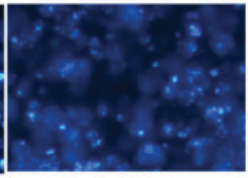

Anti-25
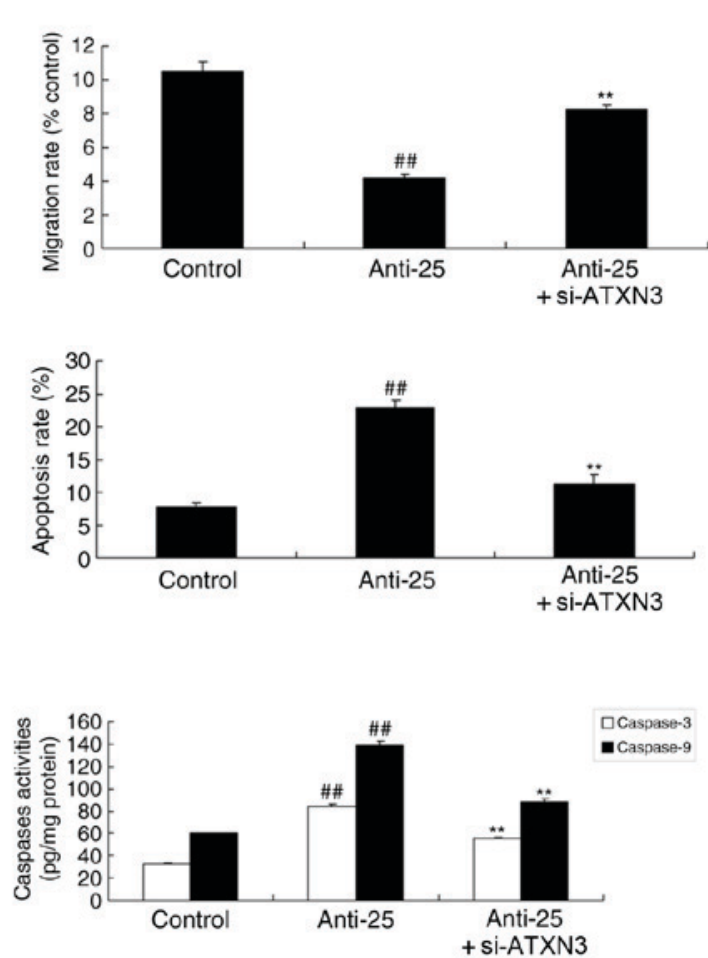

Figure 7. si-ATXN3 inhibits the anti-cancer effects of microRNA-25 downregulation on colon cancer. (A) Cell viability, (B) migration, (C) apoptosis rate and (D) caspase-3/9 activity level were determined. Data are presented as the mean \pm standard deviation. ${ }^{\# \#} \mathrm{P}<0.01$ vs. respective control; ${ }^{* *} \mathrm{P}<0.01$ vs. respective anti-25. Anti-25, microRNA-25 inhibitor; si, small interfering RNA; ATXN3, ataxin-3. 
in colon cancer cells compared with the control group (Fig. 5C-F; P<0.01). By contrast, downregulation of miR-25 significantly increased ATXN3 and Bax protein expression, and decreased cyclin D1 protein expression in colon cancer cells, compared with the control group (Fig. 5G-J; P<0.01). Based on the results, it was hypothesized that miR-25 targets ATXN3 in colon cancer cells to promote colon cancer cell growth.

si-ATXN3 inhibits the anti-cancer effects of miR-25 downregulation in colon cancer. To examine the role of ATXN3 in the anti-cancer effects of miR-25 downregulation on colon cancer, si-ATXN3 and anti-miR-25 oligos were co-transfected into colon cancer cells. Consequently, si-ATXN3 significantly decreased ATXN3 and Bax protein expression and increased cyclin D1 protein expression in colon cancer cells compared with the miR-25 downregulation group (Fig. 6). si-ATXN3 inhibited the anti-cancer effects of miR-25 downregulation on colon cancer cell proliferation and migration, apoptosis and caspase-3/9 activity level; si-ATXN3 significantly increased cell viability and migration, decreased the apoptosis rate and caspase-3/9 activity level compared with the downregulated miR-25 group (Fig. 7; $\mathrm{P}<0.01$ ).

\section{Discussion}

CRC is a glandular epithelium-derived malignant tumor and its morbidity exceeds that of gastric cancer (11). It is the most common malignant tumor in the digestive tract and its morbidity is the third highest among all cancer types globally (11). The prevalence of CRC in populations demonstrates (12). A previous study suggested that a young patient population is associated with poorer histopathological type and more dismal prognosis (12). At present, comprehensive treatment is dominated by radical surgery supplemented with radiotherapy, chemotherapy and traditional Chinese medicine treatment, with biological immunotherapy is the principal treatment for CRC (13). However, existing CRC treatments cause various inconveniences for patients in their daily life and reduces their quality of life (13). Consequently, the genesis and development mechanism of $\mathrm{CRC}$ requires elucidation and novel therapeutic targets require investigation (13). Research on miRNAs and their associations with tumors has attracted increasing attention (13). miRNAs can affect tumor cell proliferation, apoptosis, migration, angiogenesis and resistance (13). Collectively, the present results demonstrated that miR-25 expression was upregulated in patients with colon cancer, and the OS and DFS in human colon cancer with high miR-25 expression were decreased compared with in human colon cancer with low miR-25 expression. He et al (14) demonstrated that miR-25 contributes to cisplatin resistance by inhibiting forkhead box $\mathrm{O} 3 \mathrm{a}$ in gastric cancer cells.

Methylation of the ATXN3 gene results in transcription silencing, affecting the expression level of polyQ expansion mutant ataxin-2 protein (15). DNA methylation in the ATXN3 gene promoter region may partially regulate its own expression (15). Other factors, including post-transcriptional regulation, may affect the mRNA expression level (16). Therefore, DNA methylation in the promoter region may not be the principal regulatory factor affecting expression of that gene. DNA methylation is involved in regulating expression level, and also involved in pathogenesis by affecting the interaction of DNA in that region with associated DNA sequences and proteins (17). The present results suggested that overexpression of miR-25 suppressed ATXN3 protein expression in colon cancer cells. si-ATXN3 inhibited the anti-cancer effects of miR-25 downregulation in colon cancer. Huang et al (18) demonstrated that miR-25 alleviates polyQ-mediated cytotoxicity by silencing ATXN3.

Apoptosis is a gene-controlled, autonomic and programmed suicidal cell death upon stimulation to maintain homeostasis under adverse physiological conditions (19). Different from cell necrosis, apoptosis is an initiative process as opposed to a passive one. It involves the activation, expression and regulation of a series of genes, including Bax, apoptosis regulator Bcl-2, caspase-3 and poly [ADP-ribose] polymerase 1 (20). Apoptosis is not a self-damage phenomenon under pathological conditions; instead, it is a normal death process allowing cells to better adapt to the surviving environment (20). Therefore, it was hypothesized that overexpression of miR-25 suppressed Bax protein expression in colon cancer cell. Zhang et al (21) observed that miR-25 overexpression enhanced cell proliferation and reduced Bax protein expression in human ovarian cancer.

Cyclin D1 is a cell cycle-associated nucleoprotein that forms complexes with cyclin-dependent kinase (22). It serves a vital role in tumor development, and tumor proliferation and differentiation (23). Typically, cyclin D1 serves a critical role in the $G_{1}$ stage of the cell cycle, which may accelerate cell cycle progression (23). In the present study, it was identified that overexpression of miR-25 increased cyclin D1 protein expression in colon cancer cells. Huo et al (24) suggested that upregulation of miR-25 mediates the migration of melanoma cells by targeting Dickkopf-related protein 3 through effects on the expression of Myc proto-oncogene protein and cyclin D1. A limitation of the study was that only si-ATXN3 was used to downregulate the expression of ATXN3. Other mechanisms that regulate ATXN3 expression, including methylation, require investigation in future studies.

In conclusion, the present results demonstrate that miR-25 promoted human colon cancer cell growth and migration, and inhibited apoptosis via ATXN3 expression. The miR-25/ATXN3 axis provides novel insight into the pathogenesis of human colon cancer, particularly with respect to promote proliferation and metastasis of $\mathrm{CRC}$, and it represents a potential therapeutic target for human colon cancer.

\section{Acknowledgements}

Not applicable.

\section{Funding}

No funding was received.

\section{Availability of data and materials}

The datasets used and/or analyzed during the current study are available from the corresponding author on reasonable request. 


\section{Authors' contributions}

DL designed the experiments. TZ, JL, JZ, TW, YL, SH and $\mathrm{ZH}$ performed the experiments. DL and $\mathrm{TZ}$ analyzed the data. DL wrote the manuscript. All authors read and approved the final manuscript.

\section{Ethics approval and consent to participate}

The present study was approved by the Ethics Committee of the Yue Bei People's Hospital (Shaoguan, China). Written informed consent was obtained from all patients.

\section{Patient consent for publication}

Not applicable.

\section{Competing interests}

The authors declare that they have no competing interests.

\section{References}

1. Guo J, Tao W, Tang D and Zhang J: Th17/regulatory T cell imbalance in sepsis patients with multiple organ dysfunction syndrome: attenuated by high-volume hemofiltration. Int J Artif Organs 40: 607-614, 2017.

2. Zivkovic AR, Tourelle KM, Brenner T, Weigand MA, Hofer S and Schmidt K: Reduced serum cholinesterase activity indicates splenic modulation of the sterile inflammation. J Surg Res 220 275-283, 2017.

3. Wang X, Luo B, Lu Y, Pang D, Zheng J, Mo J, Huang H and Feng J: The triggering receptor expressed by myeloid cells-1 activates TLR4-MyD88-NF- $\mathrm{BB}$-dependent signaling to aggravate ventilation-induced lung inflammation and injury in mice. Cell Tissue Res 374: 137-148, 2018.

4. Li JZ, Wang ZL, Xu WH, Li Q, Gao L and Wang ZM: MicroRNA-495 regulates migration and invasion in prostate cancer cells via targeting Akt and mTOR signaling. Cancer Invest 34: 181-188, 2016.

5. Wang H, Li XT, Wu C, Wu ZW, Li YY, Yang TQ, Chen GL, Xie XS, Huang YL, Du ZW and Zhou YX: miR-132 can inhibit glioma cells invasion and migration by target MMP16 in vitro. Onco Targets Ther 8: 3211-3218, 2015.

6. Zhang D, Sun G, Zhang H, Tian J and Li Y: Long non-coding RNA ANRIL indicates a poor prognosis of cervical cancer and promotes carcinogenesis via PI3K/Akt pathways. Biomed Pharmacother 85: 511-516, 2017.

7. Yao C, Shi X, Zhang Z, Zhou S, Qian T, Wang Y, Ding F, Gu X and $\mathrm{Yu}$ B: Hypoxia-induced upregulation of miR-132 promotes schwann cell migration after sciatic nerve injury by targeting PRKAG3. Mol Neurobiol 53: 5129-5139, 2016.

8. Leinders M, Üceyler N, Pritchard RA, Sommer C and Sorkin LS: Increased miR-132-3p expression is associated with chronic neuropathic pain. Exp Neurol 283: 276-286, 2016.

9. Xu T, Pang Q, Wang Y and Yan X: Betulinic acid induces apoptosis by regulating PI3K/Akt signaling and mitochondrial pathways in human cervical cancer cells. Int J Mol Med 40: 1669-1678, 2017.
10. Livak KJ and Schmittgen TD: Analysis of relative gene expression data using real-time quantitative PCR and the 2(-Delta Delta C(T)) method. Methods 25: 402-408, 2001.

11. Yadav N and Chandra H: Suppression of inflammatory and infection responses in lung macrophages by eucalyptus oil and its constituent 1,8-cineole: Role of pattern recognition receptors TREM-1 and NLRP3, the MAP kinase regulator MKP-1, and NFKB. PLoS One 12: e0188232, 2017.

12. van Kampen JGM, van Hooij O, Jansen CF, Smit FP, van Noort PI, Schultz I, Schaapveld RQJ, Schalken JA and Verhaegh GW: miRNA-520f Reverses epithelial-to-mesenchymal transition by targeting ADAM9 and TGFBR2. Cancer Res 77: 2008-2017, 2017.

13. Liu XJ,Zheng XP,Zhang R, Guo YLand Wang JH: Combinatorial effects of miR-20a and miR-29b on neuronal apoptosis induced by spinal cord injury. Int J Clin Exp Pathol 8: 3811-3818, 2015.

14. He J, Qi H, Chen F and Cao C: MicroRNA-25 contributes to cisplatin resistance in gastric cancer cells by inhibiting forkhead box O3a. Oncol Lett 14: 6097-6102, 2017.

15. Li W, Lu M, Zhang Y, Xia D, Chen Z, Wang L, Yin N and Wang Z: Puerarin attenuates the daunorubicin-induced apoptosis of $\mathrm{H} 9 \mathrm{c} 2$ cells by activating the PI3K/Akt signaling pathway via the inhibition of Ca2+ influx. Int J Mol Med 40: 1889-1894, 2017.

16. Qiu ZK, Zhong DS, He JL, Liu X, Chen JS and Nie H: The anxiolytic-like effects of puerarin are associated with the changes of monoaminergic neurotransmitters and biosynthesis of allopregnanolone in the brain. Metab Brain Dis 33: 167-175, 2018.

17. Feng T, Zheng L, Liu F, Xu X, Mao S, Wang X, Liu J, Lu Y, Zhao W, Yu X and Tang W: Growth factor progranulin promotes tumorigenesis of cervical cancer via PI3K/Akt/mTOR signaling pathway. Oncotarget 7: 58381-58395, 2016.

18. Huang F, Zhang L, Long Z, Chen Z, Hou X, Wang C, Peng H, Wang J, Li J, Duan R, et al: miR-25 alleviates polyQ-mediated cytotoxicity by silencing ATXN3. FEBS Lett 588: 4791-4798, 2014.

19. Bahadori M, Baharara J and Amini E: Anticancer properties of chrysin on colon cancer cells, in vitro and in vivo with modulation of caspase-3, -9, Bax and Sall4. Iran J Biotechnol 14: 177-184, 2016.

20. LeBlanc H, Lawrence D, Varfolomeev E, Totpal K, Morlan J, Schow P, Fong S, Schwall R, Sinicropi D and Ashkenazi A: Tumor-cell resistance to death receptor-induced apoptosis through mutational inactivation of the proapoptotic Bcl-2 homolog Bax. Nat Med 8: 274-281, 2002.

21. Zhang H, Zuo Z, Lu X, Wang L, Wang H and Zhu Z: MiR-25 regulates apoptosis by targeting Bim in human ovarian cancer. Oncol Rep 27: 594-598, 2012.

22. Wang J, Li XM, Bai Z, Chi BX, Wei Y and Chen X: Curcumol induces cell cycle arrest in colon cancer cells via reactive oxygen species and Akt/GSK3 $\beta /$ cyclin D1 pathway. J Ethnopharmacol 210: 1-9, 2018.

23. Li C, Peng W, Song X, Wang Q and Wang W: Anticancer effect of icaritin inhibits cell growth of colon cancer through reactive oxygen species, Bcl-2 and cyclin D1/E signaling. Oncol Lett 12: 3537-3542, 2016.

24. Huo J, Zhang Y, Li R, Wang Y, Wu J and Zhang D: Upregulated MicroRNA-25 mediates the migration of melanoma cells by targeting DKK3 through the WNT/ $\beta$-catenin pathway. Int J Mol Sci 17: pii: E1124, 2016.

This work is licensed under a Creative Commons Attribution-NonCommercial-NoDerivatives 4.0 International (CC BY-NC-ND 4.0) License. 